\title{
Performance Analysis of an Adaptive Incremental Cooperative Relaying Scheme for Wireless Relay Networks
}

\author{
Shaoqing Wang, Leiming Zhang, Yuwu Yan \\ The electronic system engineering company of China \\ No. 58 Nan Fang Zhuang Jia, Fengtai District, Beijing, China \\ e-mail: 429086198@qq.com
}

\begin{abstract}
In wireless relaying networks, the traditional incremental cooperative relaying scheme (IR) could improve the system throughput enormously over fading channels by exploiting relay nodes to retransmit the source data packet to the destination. In order to enhance the system throughput over time-varying fading channels, this paper proposes an adaptive incremental cooperative relaying scheme (AIR) based on adaptive modulation and coding, which implements adaptive rate transmission for the source and relay nodes according to channel state information. We derive expressions for the AIR system throughput, and then give a gradient-based search algorithm to find the optimized adaptive solution for the AIR system by maximizing throughput subject to the constraint of packet error rate at the data link layer. The results indicate that throughput of AIR system outperforms that of traditional IR system greatly for any SNR value.
\end{abstract}

Keywords-cooperative relaying; adaptive modulation coding; throughput; packet error rate

\section{INTRODUCTION}

In wireless relay networks, an efficient way of combating channel fading is cooperative diversity in which the source node transmits information to the destination not only through a direct-link but also through the use of relays [1]-[2]. The basic idea of it results from the broadcasting property of wireless transmission so that other nodes in the same network can overhear the information from the source node and can then forward what is received to the destination. So, cooperative diversity can provide multiple independently-faded channels for the same information transmission so that the probability that all received signals at the destination suffer deep fading will be significantly reduced. A variety of low-complexity cooperative relaying scheme were proposed firstly in [3], which included fixed relaying (FR), selection relaying (SR) and incremental relaying (IR) schemes. However, both FR and SR schemes lead to a certain loss in spectral efficiency because it requires two time phase periods for half-duplex transmission. IR performs in full-duplex transmission when the direct transmission between the source and destination nodes is successful and in half-duplex transmission only when the direct transmission is not successful, so IR scheme can increase the system spectral efficiency over FR or SR scheme. More cooperative relaying schemes were studied and analyzed for various systems or channel models detailedly in [4]-[7].
However, all the aforementioned papers only consider fixed rate transmission in cooperative relaying system, which results in a deteriorated throughput performance over time-varying fading channels. Adaptive transmission at the transmitter based on adaptive modulation and coding (AMC) has been shown to be effective to enhance the system performance over time-varying fading channels ${ }^{[8]}$. To exploit the benefits of rate adaptation over the relay channel, recently, several studies have been reported in [9]-[11]. In [9], the authors proposed a discrete rate and power adaptation policy based on FR scheme for the cooperative relay networks. In [10], the authors also proposed the use of FR scheme with adaptive transmission techniques, where only the source adapts its rate according to the changing channel conditions, while the relays simply amplify and forward the signals. In [11], the authors gave a cross-layer design method which combines truncated ARQ at the link layer and adaptive cooperative diversity at the physical layer, in which both the source node and the relay nodes must utilize an orthogonal space-time block code for packet retransmission.

It is known that multimedia application not only require wireless relay networks to support the higher transmission rate, but also need the much better packet-level quality of service (QoS) performance such as packet error rate (PER) at the data link layer. However, both the packet-level QoS requirement in wireless relay networks and the effect of cooperative relaying on the higher layer aren't considered in [9]-[11]. Designing cooperative relaying schemes suitable for QoS constrained multimedia applications in wireless relay networks is still an open problem.

Motivated by these researches, in order to enhance the system throughput over time-varying fading channels, we propose an adaptive incremental decode-and-forward cooperative relaying (AIR) scheme based on AMC for the single-relay transmission system, which implements adaptive rate transmission of the source and relay nodes according to channel state information. The AIR system throughput is defined as the data rate successfully received at the destination node in this paper. We firstly derive expressions for the AIR system throughput, and then give a gradient-based search algorithm to find the optimized adaptive solution for the AIR system by maximizing throughput subject to the constraint of packet error rate at the data link layer. In addition, throughput of the traditional IR system is also deduced and compared with that of the AIR system. 
Different from researches in [9]-[11], the design objective of the AIR scheme is maximizing the system throughput while satisfying the packet-level QoS requirement (the constraint of packet error rate). Moreover, in the AIR system, both source and relay node can employ different adaptive modulation coding modes or transmission rate at physical layer and the relay node only retransmits the packet erroneously received at the destination in order to enhance the system spectral efficiency over the adaptive FR schemes proposed in [9] and [10]. At last, the AIR scheme is easier to implement than the adaptive relaying scheme based on distributed space-time codes proposed in [11], as the later requires synchronization between the spatially separated relays performing the distributed space-time code [12-13]

The remainder of this paper is organized as follows. Section II introduces the system models and gives the AIR scheme. We derive expression for AIR system throughput and present a gradient-based search algorithm to find the optimized adaptive solution in Section III. The traditional IR system Throughput is deduced in section IV. Some numerical and simulation results are given in Section V. Finally, some conclusions are drawn in section VI.

\section{SYSTEM MODEL AND ADAPTIVE INCREMENTAL RELAYING SCHEME}

packet. If $\mathrm{R}$ has received the source packet successfully, it retransmits it to the $\mathrm{D}$ and $\mathrm{D}$ drops the corrupted packets and only decodes based on the retransmitted packet. If D still fails to receive the retransmitted packet, this packet will be dropped. Moreover, if the $\mathrm{R}$ fails to receive the source packet, this packet will be also dropped.

Adopting the block fading channel model for the S-D, R-D and S-R links, the channel gains remain constant over a time slot, but vary from one time slot to another. AMC is employed for both the S-D and R-D links in order to implement the adaptive rate transmission. Based on the perfect channel state information (CSI) acquired at the D node, the AMC selector determines the AMC mode which is fed back to the $\mathrm{S}$ or $\mathrm{R}$ node through the feedback channel without error and latency. Then, the AMC controller of the $\mathrm{S}$ or $\mathrm{R}$ node updates the transmission mode and transmits the new data frame.

Let $N$ denote the total number of transmission mode available and define instantaneous SNR of the S-D and R-D links as $\gamma_{1}$ and $\gamma_{2}$ respectively. The system partitions the entire SNR range into $N+1$ nonoverlapping consecutive intervals with the SNR thresholds denoted as

$$
\left\{\gamma_{m, n}\right\}\left(\gamma_{m, 0}=0, \gamma_{m, N+1}=\infty\right)
$$

where $m=1,2$ refers to the S-D and R-D links respectively and $n=0,1, \ldots, N$ is the mode index.

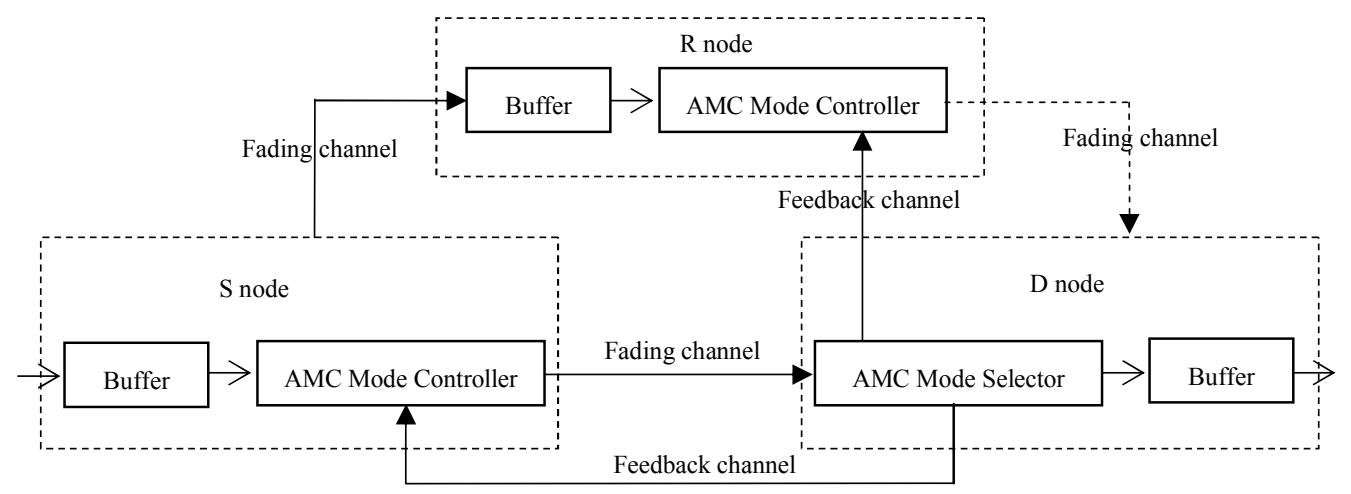

Figure 1. System model

Consider three relevant nodes in a wireless relay network, represented respectively by $\mathrm{S}$ (source node), $\mathrm{R}$ (relay node) and $\mathrm{D}$ (destination node), and assume that $\mathrm{S}$ wants to sent data to $\mathrm{D}$, as illustrated in figure 1 . At $\mathrm{S}$, packets are stored in a transmit buffer, grouped into frames, and then transmitted over the wireless channel on a frame by frame basis. Assume that the cyclic redundancy check (CRC) bits of each packet facilitate perfect error detection at the receiving nodes. The time is slotted, and the length of a time slot can satisfy the transmission latency and spread latency of the data frame. The proposed AIR scheme acts as follows. $S$ node firstly transmits a data frame in the current time slot. For one case, upon successful reception of all packets in this frame at D, it sends back an ACK message, then S transmits a new data frame in the next time slot and the $\mathrm{R}$ node just idles. For the other case, if the $\mathrm{D}$ receives a packet in error, it broadcasts a NACK message identifying the corrupted
Specifically, mode $n$ is chosen for the S node when SNR

$$
\gamma_{1} \in\left[\gamma_{1, n}, \gamma_{1, n+1}\right)
$$

Then the $\mathrm{S}$ node transmits the data frame in the corresponding rate $R_{n}$ bit/symbol.

Similarly, when SNR

$$
\gamma_{2} \in\left[\gamma_{2, n}, \gamma_{2, n+1}\right)
$$

$\mathrm{R}$ node retransmits the corrupted packet to $\mathrm{D}$ node in the rate $R_{n-1}$ bits/symbol.

To avoid deep channel fades, no data frame will be sent when $\gamma_{m}<\gamma_{m, 0}$.

If the symbol rate of the system is constant, $R_{n}$ can stand for the data rate. We define throughput of the AIR system as the average data rate successfully received at the D node, so throughput can be expressed by 


$$
T=E\left[R_{n}\right] \frac{L-C}{L}\left(1-P E R_{a}\right)
$$

where $E[]$ stands for expected operation, $E\left[R_{n}\right]$ represents the expected data rate, $L$ is the length of one packet, $C$ is the length of CRC bits and $P E R_{a}$ is the average packet error rate (PER) of the system. To derive expression of throughput, we need to deduce expressions of the average PER and expected data rate respectively in the next section.

\section{PERFormance ANALYSIS}

\section{A. The average packet error rate}

Let $P E R_{S D-a}, P E R_{R D-a}$ and $P E R_{S R-a}$ denote the average PER of the S-D, R-D and S-R links respectively, so the average PER of the system can be expressed by

$$
\begin{gathered}
P E R_{a}=P E R_{S D-a} P E R_{S R-a}+P E R_{S D-a} \square \\
\left(1-P E R_{S R-a}\right)\left(P E R_{R D-a}\right)
\end{gathered}
$$

where the first term corresponds to the event that the source packet is received in error at both the $\mathrm{D}$ and $\mathrm{R}$ nodes and the second term corresponds to the event that the packet is received in error at the $D$ node but successfully at the $R$ nodes, and the $\mathrm{R}$ node's retransmission to the $\mathrm{D}$ node is still in error. The expressions of $P E R_{S D-a}, P E R_{R D-a}$ and $P E R_{S R-a}$ will be deduced in the following.

All the channels are modeled as a Rayleigh block fading channel and AWGN with variance $N_{0}$. Assume that the transmit power is constant for all nodes, denoted by $P_{t}, d_{1}$, $d_{2}$ and $d_{3}$ denote the distance of S-D, R-D, and S-R links respectively and path loss exponent is represented by $\alpha$, so the probability distribution function (PDF) of the received SNR for the S-D and R-D links can be written as respectively

$$
p_{\gamma_{m}}(\gamma)=\frac{1}{\overline{\gamma_{m}}} \exp \left(-\frac{\gamma}{\overline{\gamma_{m}}}\right)
$$

where $m=1,2,3$ refers to the S-D, R-D and S-R links respectively and $\overline{\gamma_{1}}=P_{t}\left(d_{1}\right)^{-\alpha} / N_{0}, \overline{\gamma_{2}}=P_{t}\left(d_{2}\right)^{-\alpha} / N_{0}$ and $\overline{\gamma_{3}}=$ $P_{t}\left(d_{3}\right)^{-\alpha} / N_{0}$ stand for the average received SNR of links.

According to the AMC rule in (2) and (3), each mode $n$ for the $\mathrm{S}$ and $\mathrm{R}$ nodes will be chosen with probability

$$
p_{m, n}=\int_{\gamma_{m, n}}^{\gamma_{m, n+1}} p_{\gamma_{m}}(\gamma) d \gamma_{m}(m=1,2)
$$

Noting that the instantaneous PER depends on the received SNR $\gamma$, in order to simplify the analysis, we approximate the instantaneous PER for the AMC mode $n$ as [8]

$$
\operatorname{PER}_{n}(\gamma)=\left\{\begin{array}{cc}
1, & 0<\gamma<\gamma_{p n} \\
a_{n} \exp \left(-g_{n} \gamma\right), & \gamma>\gamma_{p n}
\end{array}\right.
$$

where the parameters $\left\{a_{n}, g_{n}, \gamma_{p n}\right\}$ are obtained by fitting (8) to the exact PER of mode $n$.

Let $P E R_{m, n}$ denote the average PER for mode $n$. Combining (6), (7) and (8), the average PER of the S-D and R-D links for mode $n$ can be written as

$$
\operatorname{PER}_{m, n}=\frac{1}{p_{m, n}} \int_{\gamma_{m, n}}^{\gamma_{m, n+1}} \operatorname{PER}_{n}(\gamma) p_{\gamma_{m}}(\gamma) d \gamma_{m}
$$

where $m=1,2$ refers to the S-D and R-D links respectively.

So, the PER of the S-R link for mode $n$ can be expressed by

$$
\operatorname{PER}_{3, n}=\frac{1}{p_{1, n}} \int_{0}^{\infty} \operatorname{PER}_{n}(\gamma) p_{\gamma_{3}}(\gamma) d \gamma_{3}
$$

The average PER of links can be computed as the ratio of the average number of incorrectly received packets over the total average number of transmitted packets. So, we have

$$
\begin{aligned}
\operatorname{PER}_{S D-a}= & \frac{\sum_{n=1}^{N} R_{n} p_{1, n} P E R_{1, n}}{\sum_{n=1}^{N} R_{n} p_{1, n}} \\
\operatorname{PER}_{R D-a}= & \frac{\sum_{n=1}^{N} R_{n} p_{2, n} P E R_{2, n}}{\sum_{n=1}^{N} R_{n} p_{2, n}} \\
\operatorname{PER}_{S R-a}= & \frac{\sum_{n=1}^{N} R_{n} p_{1, n} P E R_{3, n}}{\sum_{n=1}^{N} R_{n} p_{1, n}}
\end{aligned}
$$

Substituting expression (11), (12) and (13) into (5), we can derive expression of the average PER of the system.

\section{B. The expected data rate}

We define the expected data rate $E\left[R_{n}\right]$ as the average number of accepted information bits per symbol at the $D$ node. Let $R_{1, n}$ denotes that $\mathrm{S}$ node transmits data packets with data rate $R_{n}$ and $R_{2, n}$ denotes that $\mathrm{R}$ node retransmits the corrupted data packet with data rate $R_{n}$, so, the expected data rate can be expressed as

$$
\begin{aligned}
E\left[R_{n}\right]= & E\left[R_{1, n}\left(\left(1-\operatorname{PER}_{n}\left(\gamma_{1}\right)\right)+\operatorname{PER}_{n}\left(\gamma_{1}\right) P E R_{3, n}\right)\right]+ \\
& E\left[\frac{L}{\frac{L}{R_{1, n}}+\frac{L}{R_{2, n}}} \operatorname{PER}_{n}\left(\gamma_{1}\right)\left(1-\operatorname{PER}_{3, n}\right)\right]
\end{aligned}
$$

where the first term corresponds to the event that a packet transmitted by the $\mathrm{S}$ node with data rate $R_{1, n}$ is received successfully by $\mathrm{D}$ or received erroneously by both the $\mathrm{D}$ and $\mathrm{R}$ nodes and the second term corresponds to the event that the $\mathrm{R}$ node retransmit the corrupted packet with data rate $R_{2, n}$.

Considering that the total number of AMC modes is $N$, using (6) and (14), (14) can be rewritten as 


$$
\begin{aligned}
E\left[R_{n}\right]= & \sum_{n=1}^{N} \int_{\gamma_{1, n}}^{\gamma_{1, n+1}} R_{1, n}\left(\left(1-P E R_{n}\left(\gamma_{1}\right)\right)+\operatorname{PER}_{n}\left(\gamma_{1}\right) P E R_{3, n}\right) \\
& p_{\gamma_{1}}(\gamma) d \gamma_{1}+\sum_{n=1}^{N} \sum_{n=1}^{N} \int_{\gamma_{1, n}}^{\gamma_{1, n+1}} \int_{\gamma_{2, n}}^{\gamma_{2, n+1}} \frac{R_{1, n} R_{2, n}}{R_{1, n}+R_{2, n}} P E R_{n}\left(\gamma_{1}\right) \square \\
& \left(1-P E R_{3, n}\right) p_{\gamma_{1}}(\gamma) p_{\gamma_{2}}(\gamma) d \gamma_{1} d \gamma_{2}
\end{aligned}
$$

\section{The optimum of throughput}

Substituting (5) and (15) into (4), we can derive expression of the system throughput. The design objective of the AIR scheme is to maximize the system throughput, while satisfying the packet error rate requirement. So, we need to determine the optimized SNR thresholds $\left\{\gamma_{m, n}\right\}$ for the AMC mode of AIR system in order to maximize system throughput.

So, the desired optimized problem can be formulated as follows

$$
\begin{gathered}
\max _{\left\{\gamma_{1, n}, \gamma_{2, n}\right\}} T_{A I R} \\
\text { s.t. } \quad P E R_{a} \leq P E R_{t}
\end{gathered}
$$

which means that the average PER of the system is not greater than the threshold of PER $\left(P E R_{t}\right)$.

Firstly, we formulate the total PER constraint in (16) into two separate PER constraints for the S-D and R-D links as follows

$$
\begin{aligned}
& P E R_{1, n} \leq P E R_{S D-t} \\
& P E R_{2, n} \leq P E R_{R D-t}
\end{aligned}
$$

where $P E R_{S D-t}$ and $P E R_{R D-t}$ are the threshold of PER for the $\mathrm{S}-\mathrm{D}$ and R-D links respectively.

Combing (5), (16), (17) and (18), we have

$$
\begin{gathered}
P E R_{S D-t}\left(P E R_{S R-a}+\left(1-P E R_{S R-a}\right)\left(P E R_{R D-t}\right)\right) \leq P E R_{t} \\
P E R_{R D-t}=\frac{\frac{P E R_{t}}{P E R_{S D-t}}-P E R_{S R-a}}{\left(1-P E R_{S R-a}\right)}
\end{gathered}
$$

Using (8) and (17), we can derive expressions of the SNR threshold of AMC mode for the S-D link as follows

$$
\gamma_{1, n}=\max \left\{\frac{1}{g_{n}} \ln \left(a_{n} / P E R_{S D-t}\right), \gamma_{p n}\right\}
$$

Combining (8) and (20), we can derive expressions of the SNR threshold of AMC mode for the R-D link as follows

$$
\gamma_{2, n}=\max \left\{\frac{1}{g_{n}} \ln \left(\frac{a_{n}\left(1-P E R_{S R-a}\right)}{\frac{P E R_{t}}{P E R_{S D-t}}-P E R_{S R-a}}\right), \gamma_{p n}\right\}
$$

Using (16), (21) and (22), the optimized problem in (16) can be rewritten as

$$
\begin{array}{cc} 
& \max _{P E R_{S D-t}} T_{A I R} \\
\text { s.t. } & P E R_{t} \leq P E R_{S D-t} \leq 1
\end{array}
$$

In the following, we give a gradient-based search algorithm to find the optimized the SNR threshold of AMC mode. Let $P E R_{S D-t}=M, T(M)$ denotes the corresponding throughput, $\partial T(M) / \partial M$ is the derivative of $T(M), M_{o}$ represents the optimum value of $M$ and $T_{\max }$ denotes the corresponding maximal value of throughput. Moreover, let $\varepsilon$ to be a finite small positive real number.

The pseudo code of the gradient-based search algorithm is as follows.

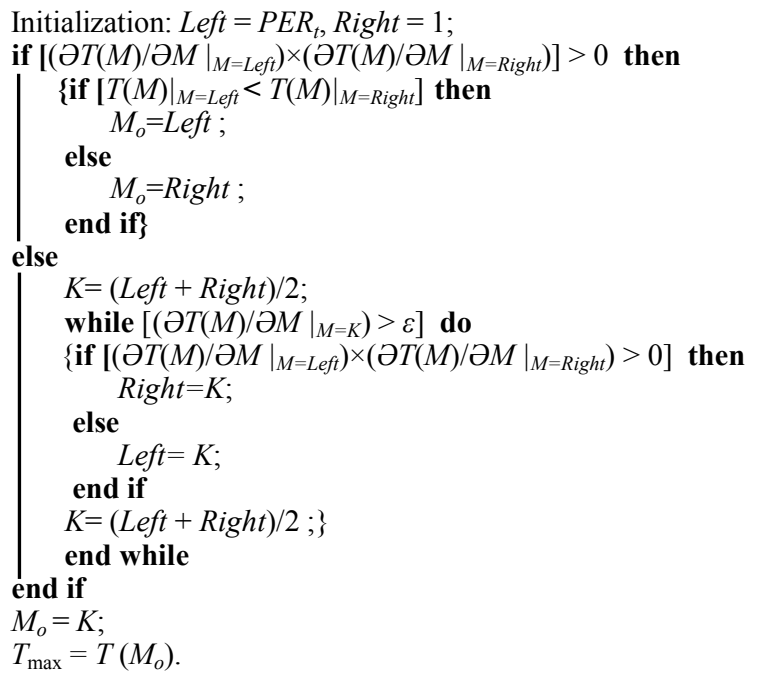

\section{Throughrut OF THE CONSTANT RATE INCREMENTAL RELAY (CRIR) SYSTEM}

In this section, we deduce expression of the CRIR system throughput to compare it with the AIR system throughput.

Similarly to the AIR system, the CRIR system throughput can be expressed by

$$
T_{C R I R}=R_{C R I R} \frac{L-C}{L}\left(1-P E R_{C R I R}\right)
$$

where $R_{C R I R}$ denotes the expected data rate and $P E R_{C R I R}$ is the average packet error rate of CRIR system.

Similarly to (5), the average PER of CRIR system can be written as

$P E R_{C R I R}=P E R_{S D} \cdot P E R_{S R}+P E R_{S D}\left(1-P E R_{S R}\right) P E R_{R D}(25)$ where $P E R_{S D}, P E R_{S R}$ and $P E R_{R D}$ is the average PER of S-D, $\mathrm{S}-\mathrm{R}$ and R-D links respectively and can be expressed as follows

$$
\begin{aligned}
\operatorname{PER}_{S D} & =\int_{0}^{\infty} P E R_{n}\left(\gamma_{1}\right) p_{\gamma_{1}}(\gamma) d \gamma_{1} \\
\operatorname{PER}_{S R} & =\int_{0}^{\infty} P E R_{n}\left(\gamma_{3}\right) p_{\gamma_{3}}(\gamma) d \gamma_{3} \\
P_{R D} & =\int_{0}^{\infty} P E R_{n}\left(\gamma_{2}\right) p_{\gamma_{2}}(\gamma) d \gamma_{2}
\end{aligned}
$$

The expected data rate of CRIR system can be expressed by 


$$
R_{C R I R}=\frac{\left(1-P E R_{S D}+P E R_{S D} P E R_{S R}\right)}{\frac{1}{R_{n}}}+\frac{P E R_{S D}\left(1-P E R_{S R}\right)}{\frac{1}{R_{n}}+\frac{1}{R_{n}}}
$$

where $n$ is the modulation and coding mode index.

\section{NUMERICAL AND SIMULATION RESULTS}

In this section, we provide results to compare the performance of AIR system with that of CRIR system. Without the loss of the generality, we assume that the average SNR of the S-R link is $25 \mathrm{~dB}$ and the S-D link and the R-D link have the same average SNR. In addition, transmit power $P_{t}$ is normalized to unity. In the simulation, we use the AMC mode of HYPERLAN/2 standard with packet length $L=1080$ bits as given in table $I^{[8]}$ and mode 4 in table I is employed by CRIR system.

TABLE I. TRANSMisSiON MODES WITH CODED MODULATION

\begin{tabular}{|c|c|c|c|c|c|}
\hline & Mode 1 & Mode 2 & Mode 3 & Mode 4 & Mode 5 \\
\hline Modulation & BPSK & QPSK & QPSK & 16 QAM & $\begin{array}{c}64 \\
\text { QAM }\end{array}$ \\
\hline Code rate & $1 / 2$ & $1 / 2$ & $3 / 4$ & $3 / 4$ & $3 / 4$ \\
\hline $\begin{array}{c}R_{n} \\
\text { (bit/symbol) }\end{array}$ & 0.5 & 1 & 1.5 & 3 & 4.5 \\
\hline$a_{n}$ & 274.7229 & 90.2514 & 67.6181 & 53.3987 & 35.3508 \\
\hline$g_{n}$ & 7.9932 & 3.4998 & 1.6883 & 0.3756 & 0.0900 \\
\hline$\gamma_{p n}(\mathrm{~dB})$ & -1.5331 & 1.0942 & 3.9722 & 10.2488 & 15.9784 \\
\hline
\end{tabular}

Figure 2 shows the average PER performances of the AIR and CRIR system. From the figure, we can see that AIR system performance outperforms the CRIR system for any SNR value. This is because that AMC is introduced in AIR system, in which no data frame will be sent in order to avoid deep channel fades. Moreover, the average PER of the AIR system is below the threshold of PER at the data link layer $\left(P E R_{t}=0.01\right)$ and the packet-level QoS requirement of the system is satisfied.

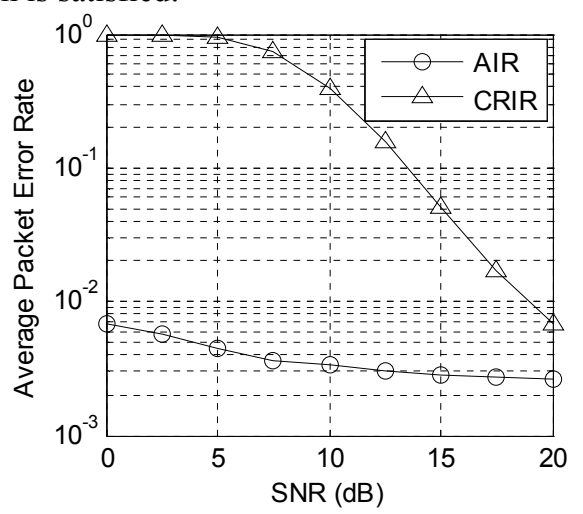

Figure 2. The average PER of AIR system vs, the average PER of CRIR system $\left(P E R_{t}=0.01\right)$

As shown in Figure 3, the AIR system throughput is a concave function of the PER threshold of S-D link and there are different optimized throughput values of AIR system for different average SNR of S-D link. The optimized throughput value of AIR system becomes larger and larger as the SNR increases. So, the gradient-based search algorithm proposed in section III. 3 can find the optimized throughput.

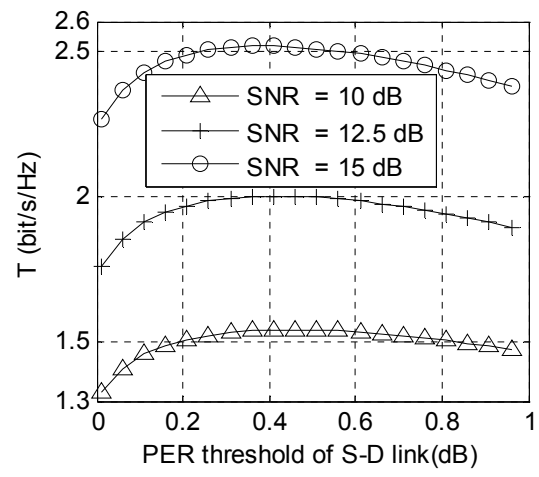

Figure 3. Throughput of AIR system vs. the threshold PER of S-D link $\left(P E R_{t}=0.01\right)$

Figure 4 compares the throughput of AIR system with that of CRIR system. Due to AMC introduced in AIR scheme, throughput of AIR system exceeds that of CRIR system for any SNR value. The simulation results are very close to the theory results, which verify the theory analysis in section III and IV.

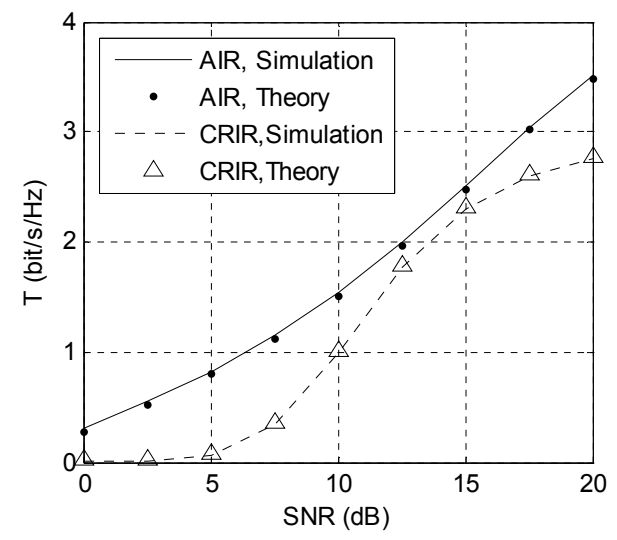

Figure 4. Throughput of AIR system vs. throughput of CRIR system $\left(P E R_{t}=0.01\right)$

\section{CONCLUSIONS AND FutuRE WORK}

In this paper, we propose an adaptive incremental cooperative relaying (AIR) scheme in order to enhance throughput of the cooperative relaying system. We derive expression for the AIR throughput and give a gradient-based search algorithm to find the optimized adaptive solution for AIR system by maximizing throughput subject to the constraint of PER at the data link layer. The numerical and simulation results indicate that throughput of AIR system outperforms that of traditional incremental relaying system greatly for any SNR value. The AIR scheme proposed in the paper establishes the theory foundation for the QoS constrained multimedia applications in wireless relay networks. 
We plan to extend our work to the multi-relay selection scenario. If there are multiple relays nodes chosen for the source node, one can select the best relay to retransmit the packet erroneously received by the destination node according to the corresponding relay selection standard such as the best harmonic mean selection method ${ }^{[12]}$. Moreover, the constant transmit power is adopted at all the nodes in this paper. In future, the power control scheme can be integrated into our research.

\section{REFERENCES}

[1] A. Sendonaris, E. Erkip, and B. Aazhang, "User cooperation diversity-part I: system description", IEEE Trans. Commun., vol. 51, no. 11, pp. 1927-1938, Nov. 2003.

[2] A. Sendonaris, E. Erkip, and B. Aazhang, "User cooperation diversity-part II: implementation aspects and performance analysis", IEEE Trans. Commun., vol. 51, no. 11, pp. 1939-1948, Nov. 2003.

[3] J. N. Laneman, G. W. Wornell, and D. N. C. Tse, "Cooperative diversity in wireless networks: efficient protocols and outage behavior", IEEE Trans. Inform. Theory, vol. 50, no. 12, pp. 30623080, Dec. 2004.

[4] A. Bletsas, A. Khisti, D. P. Reed, and A. Lippman, "A simple cooperative diversity method based on network path selection", IEEE J. Select. Areas Commun., vol. 24, no. 3, pp. 659-672, Mar. 2006.

[5] S. Ikki and M. H. Ahmed, "Performance analysis of cooperative diversity wireless networks over Nakagami-m fading channel", IEEE Commun. Lett., vol. 11, no. 7, pp. 334-336, July 2007.
[6] J. Cai, A. S. Alfa, P. Ren, X. Shen, and J. W. Mark, "Packet level performance analysis in wireless user-relaying networks", IEEE Trans. Wireless Commun., vol. 7, no. 12, pp. 5336-5345, Dec. 2008.

[7] S. S. Ikki and M. H. Ahmed, "Performance of cooperative diversity using equal gain combining (EGC) over Nakagami-m fading channels", IEEE Trans. Wireless Commun., vol. 8, no. 2, pp. 557-562, Feb. 2009.

[8] Q. Liu, S. Zhou, and G. Giannakis, "Queuing with adaptive modulation and coding over wireless links: cross-layer analysis and design”, IEEE Trans. Wireless Commun., vol. 4, no. 5, pp. 1142-1152, May 2005.

[9] N. Ahmed and B. Aazhang, "Throughput gains using rate and power control in cooperative relay networks ," IEEE Trans. Commun., vol. 55, no. 4, pp. 645-656, Apr. 2007.

[10] L. Dai and K. B. Letaief, "Throughput maximization of ad-hoc wireless networks using adaptive cooperative diversity and truncated ARQ”, IEEE Trans. Commun., vol. 56, no. 11, pp. 1907-1918, Nov. 2008.

[11] T. Nechiporenko, K. T. Phan, C. Tellambura, and H. H. Nguyen, "On the capacity of Rayleigh fading cooperative systems under adaptive transmission", IEEE Trans. on Wireless Commun., vol. 8, no. 4, pp. 1626-1631, Apr. 2009.

[12] J. N. Laneman and G. W. Wornell, "Distributed space-time-coded protocols for exploiting cooperative diversity in wireless networks", IEEE Trans. Inform. Theory, vol. 49, no. 10, pp. 2415-2425, Oct 2003.

[13] K. J. R. Liu, A. K. Sadek, W. Su, and A. Kwasinski. Cooperative Communications and Networking, Cambridge, U. K. Cambridge University Press, 2008.

[14] A. S. Ibrahim, A. K. Sadek, W. Su, and K. J. R. Liu, "Cooperative communications with relay-selection: When to cooperate and whom to cooperate with? ", IEEE Trans. on Wireless Commun., vol. 7, no. 7, pp. 2814-2827, July 2008 . 